\title{
Modeling a Porous Region for Natural Convection Heat Transfer and Experimental Validation in Slender Cylindrical Geometries
}

\author{
Olugbenga O. Noah, * Johan F. Slabber, and Josua P. Meyer \\ University of Pretoria, Department of Mechanical and Aeronautical Engineering, Private Bag X20, Hatfield \\ 0028, Pretoria, South Africa
}

Received May 7, 2015

Accepted for Publication July 25, 2015

http://dx.doi.org/10.13182/NT15-56

\begin{abstract}
Natural convection heat transfer in fluid-saturated porous media has in recent years gained considerable attention especially in high-temperature reactors. It is proposed in this study that light water reactors (LWRs) can be made safer by redesigning the fuel in the fuel assembly. The proposed design is aimed at increasing the safety level in LWRs by the use of fuel in the form of loose coated particles in a helium environment inside the nuclear fuel cladding tubes of the fuel elements. The coated particle fuel being a heat source forms a bed in the cladding tube closed at both ends, the heat from the particles is transferred to the gas in the tube, and the gas movement is due to natural convection. In this study, we investigate the heat transfer characteristics inside a cladding tube containing packed beds of spherical particles by simulating a porous region whose medium properties are defined; that is, the geometrical model representing the packed bed is specified as a porous region. The finite volume method was used in solving the three-dimensional Navier-Stokes equation while the heat transfer coefficient $h$ and the dimensionless numbers such as $\mathrm{Ra}=f(\mathrm{Gr}, \mathrm{Pr})$ and $\mathrm{Nu}$ are used in analyzing the results. Simulated results from this investigation were validated with experimental results. The discrepancy in the results may be due to uncertainties, experimental errors, numerical errors, and the consequence of the lump parameter effect in the porous region modeling approach. This approach may be considered a unique means of estimating heat transfer characteristics in porous media.
\end{abstract}

Keywords - Light water reactor, porous region, heat transfer characteristics.

Note - Some figures may be in color only in the electronic version.

\section{INTRODUCTION}

Heat transfer in packed bed systems is an important operation in high-temperature nuclear reactor designs. A number of high-temperature reactors (HTRs) were developed with embedded fuel in graphite moderators randomly packed as fuel spheres in the core with helium gas as coolant flowing under the action of forced convection heat transfer around the spheres.

The heat transfer coefficient is an important parameter in the determination of the heat transfer performance in packed beds. ${ }^{1,2}$ Considerable efforts through the use of

*E-mail: Noah.Olugbenga@tuks.co.za various experimental and theoretical techniques under either steady-state or unsteady-state conditions have been made to evaluate the heat transfer coefficient in fixed bed reactors. $^{3-5}$ Although much information is available for forced convection heat transfer in packed beds ${ }^{6}$ under supercritical conditions, there is very little information on heat transfer in packed beds under natural convection.

It is proposed in this study that light water reactors can be made safer by redesigning the fuel in the fuel assembly. Experimental and theoretical study of fluid-toparticle heat transfer was carried out under natural convection to better understand particle-to-fluid heat transfer characteristics expected in the proposed new fuel design. Knowledge gained through the study has assisted in 
establishing a theoretical relation required for particle-tofluid heat transfer performance in the cladding tube for the proposed design.

In recent years, the use of numerical simulation through computational fluid dynamics (CFD) offers the opportunity to predict the heat transfer and fluid flow phenomena thereby providing important gain in time, limiting the number of experiments, and accessing information at a large scale that may not be measurable with experimental methods, and it is also possible to take into account real physical properties such as high temperature and pressure conditions. ${ }^{7}$ Two approaches can be adopted in simulating porous media. The first is the traditional method; it is a direct simulation of spherical packing permeated by fine-scale voids that permit the passage of fluids in a cylindrical vessel. Direct simulation of packed beds randomly arranged through the use of the discrete element method approach is effective when studying the characteristics of contacting particles interacting with each other and quantifying the properties of the porous medium. ${ }^{8}$ The number of particles in the medium that can be simulated using this approach is limited due to the large computing capacity required. ${ }^{9}$ The second is by simulating a porous region whose medium properties are defined; typically, the geometry model representing the packed bed is specified as a porous region. In many CFD simulations involving packing materials (bed particles), it is not the detail of the internal flow that is of interest but rather the macroscopic effect of the porous medium on the overall fluid flow and heat transfer. ${ }^{10}$

This paper presents the use of the second simulation approach in validating the heat transfer characteristics in a porous medium experimental investigation. The goal of the study is to evaluate the validation and suggest if the porous region modeling approach can adequately predict the heat transfer characteristics in the proposed new fuel design contained in a cladding considering the lumped parameter effect in the approach and other uncertainties. This approach has rarely been used; hence, its investigation is worthwhile.

\section{EXPERIMENTAL SETUP}

The schematic diagram and experimental setup for this investigation are displayed in Figs. 1a and 1c. The setup consists of a cylindrical enclosure containing stainless steel (ANSI 304) balls (particles); the cylinder is manufactured from a highly insulating solid PTFE (polytetrafluoroethylene) material. The setup is made up of a cylindrical container placed on an induction heater, pressure transducers with transmitters, sets of calibrated T-type thermocouples connected to a data logger, a helium gas cylinder, a computer system, and some other accessories. A PolyScience Circulating Bath PD20R-30$\mathrm{A} 12 \mathrm{E}$ was used for performing the calibration of the T-type thermocouples used in this experimental study before the test particles and particle test sample were instrumented with the thermocouples. The calibration is carried out to determine the error reading associated with each thermocouple. The readout of the thermocouples is compared to the readout of a secondary standard at the same conditions. Using regression, a slope and intercept are determined in order to adjust by appropriate difference to reflect the same readout as the secondary; this is carried out to ensure accurate data measurement in the experiment. The 34970A Agilent Data Acquisition/Switch Unit is used as a data logger and is connected to a computer system to acquire data (see Fig. 1c). It makes use of three sets of 20-channel Armature Multiplexer cards onto which the thermocouples and pressure transducers are connected. The pressure transducers used in the experiments are calibrated to secondary standards as well.

The container has removable top and bottom lids, an inductively heated bottom steel plate lying on the inside floor of the bottom lid, an inlet valve fixed to the cylinder wall, and a pressure relief valve fitted to the top lid; a wire mesh for suspending the bed in the tube to allow for convectional fluid flow current was placed a little distance vertically above the bottom plate inside the cylinder. The particles were poured randomly into the cylindrical container; nine selected particles were instrumented with thermocouples in such a way as to measure temperatures at the surface and central point inside the particle.

Thermocouples were also placed in the interstices to measure the average gas temperature close to these particles; silicon tubes connected to pressure transducers were placed below and above the bed to measure possible pressure drop across the bed. The air in the cylinder was initially flushed out before the cylinder was later finally filled with helium gas and sealed off by the inlet valve. Thermal energy enters the packed bed by means of the inductively heated steel plate inside the cylinder that transfers heat to the helium gas immediately above the plate. Buoyancy-driven convection flow then takes place in the medium. The particle test sample was raised to $\sim 450 \mathrm{~K}$ by the heated helium gas taken at $\sim 6 \mathrm{~h}$. Thermomechanical properties of the particles are taken directly from tables for an ANSI 304-type stainless steel sphere at room temperature (see Table I). Data were collected using a data logger connected to a computer system. The data collected were analyzed before application in theoretical formulations developed for determining the heat transfer phenomenon. 


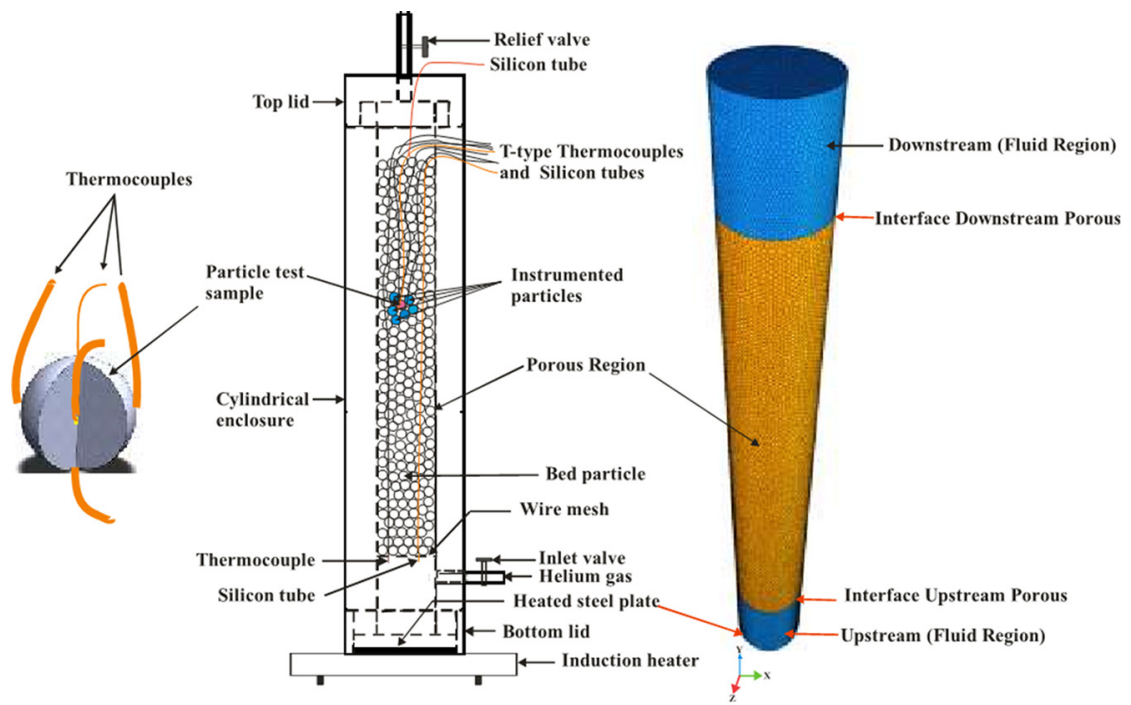

(a)

(b)

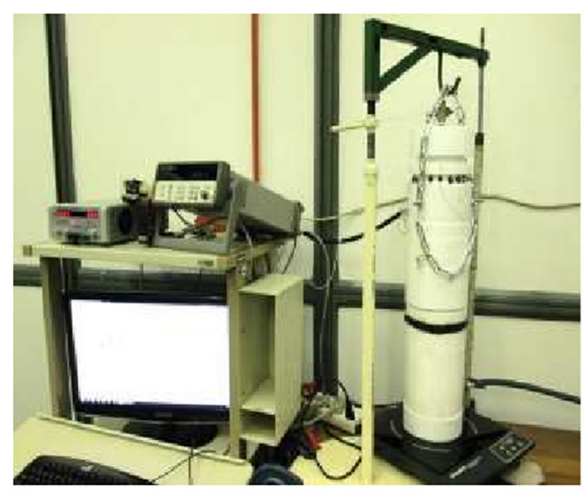

(c)

Fig. 1. (a) Schematic diagram of experimental setup (for illustrative purposes). (b) Meshed geometry model diagram of experimental setup boundary face wall for particle generating heat source porous medium. (c) Experimental setup.

TABLE I

Parameters Used in Determining the Heat Transfer Effect in the Medium

\begin{tabular}{|l|r|}
\hline \multicolumn{1}{|c|}{ Parameter } & \multicolumn{1}{|c|}{ Value } \\
\hline Density, $\rho_{p}\left(\mathrm{~kg} / \mathrm{m}^{3}\right)$ & 7913 \\
Poisson ratio, $v$ & 0.29 \\
Young's modulus, $E(\mathrm{GPa})$ & 193 \\
Particle radius, $\mathrm{r}_{p}(\mathrm{~mm})$ & 8.73 \\
Thermal diffusivity, $\alpha\left(\mathrm{m}^{2} / \mathrm{s}\right)$ & $10^{-6}$ \\
Thermal conductivity, $k_{p}(\mathrm{~W} / \mathrm{m} \cdot \mathrm{K})$ & 15.6 \\
Number of particles & 410 \\
Prandtl number, Pr & 0.689 \\
Specific heat ratio of gas, $\gamma_{g}$ & 1.667 \\
Molecular mean free part, $\Lambda(\mathrm{nm})$ & 173.6 \\
Thermal accommodation coefficient & 0.55 \\
Initial enclosed cavity pressure $(\mathrm{abs})(\mathrm{kPa})$ & 86.6 \\
Induction Cooktop Power $(\max )(\mathrm{W})$ & 1800 \\
\hline
\end{tabular}

\section{MESH DESIGN AND CFD MODELING OF A POROUS REGION}

\section{III.A. Geometrical Model and Mesh Design}

The three-dimensional (3-D) computer-aided design geometrical model used in this simulation is a cylindrical enclosure representing the upstream fluid region where the heated bottom plate is located, the porous region, and the downstream fluid region (see Fig. 1b). The detailed drawing of the experimental setup prepared in Solidworks was imported into the commercial STAR-CCM+ package where surface repair was carried out on the two in-place boundary interfaces (see Fig. 2) to allow surface and volume meshing to proceed correctly due to the close proximity of neighboring boundaries. An in-place interface was required to link together two different regions (fluid and porous) by imprinting two boundaries on one another so that 


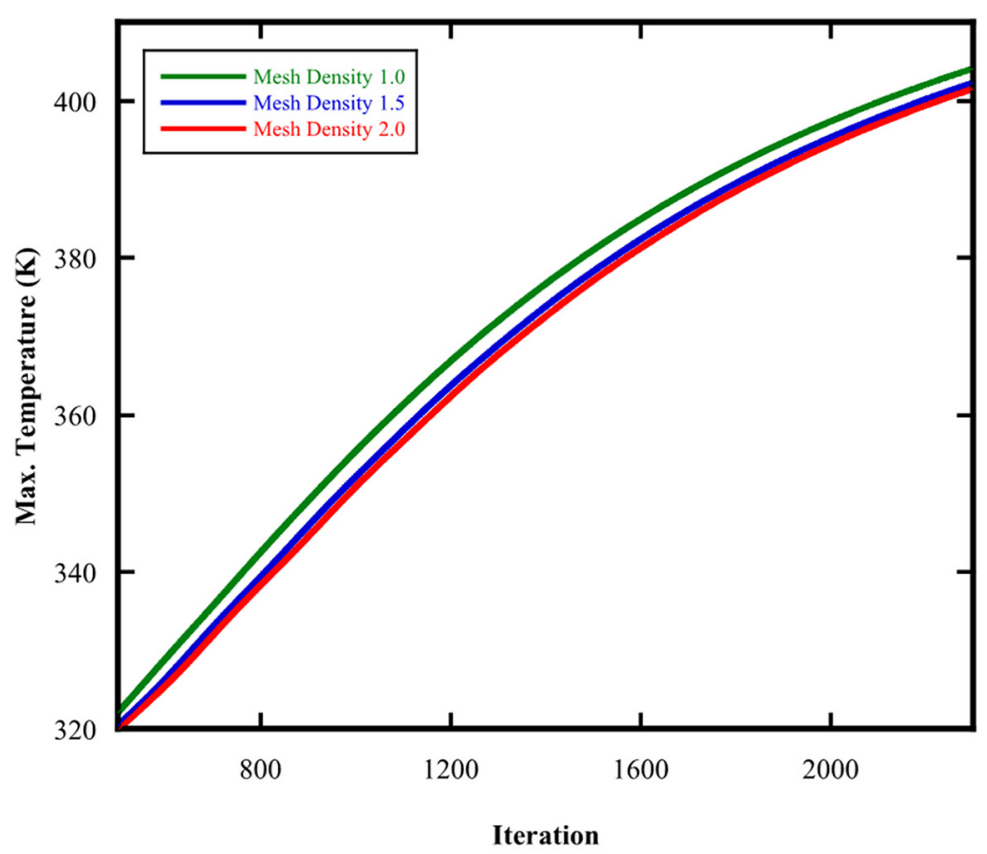

(a)

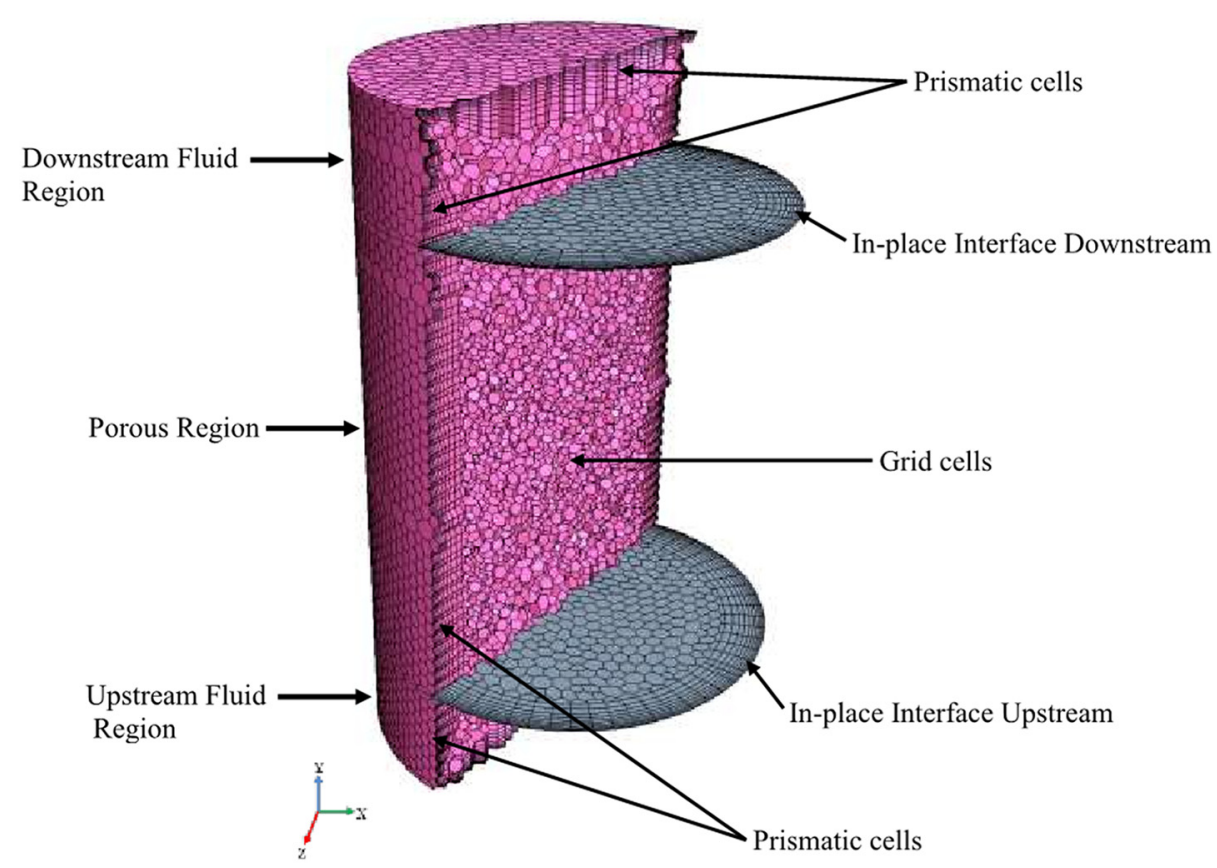

(b)

Fig. 2. (a) Mesh sensitivity analysis. (b) Threshold of a generated quality mesh of experimental model.

a conformal mesh is created at the common interface planes during the surface and volume meshing process.

A mesh is the discretized representation of the computational volume, which the physics solvers use to provide a numerical solution. STAR-CCM+ provides meshers and tools that are used to generate a quality mesh (as can be seen in Fig. 2) for various geometries and applications. Mesh quality does influence the quality of the numerical results, regardless of the setup. On a mesh of given quality and sufficient fineness, higher-order schemes yield more accurate results than a lower-order scheme. Grid skewing is an important contributing factor (much more than grid stretching) to the loss in nominal 
accuracy of the solution. Grid design (distribution of cell size, local refinement using feature edges, boundary region, or volume shapes) is important in maximizing the accuracy for a given effort. When solving steady-state problems on fine meshes, start with a much coarser mesh and then successively refine the mesh by increasing the mesh density; refinement enables better resolution of the flow. Meshes should be well designed to resolve important fluid flow features that are dependent upon flow condition parameters such as the grid refinement inside the wall boundary layer.

Grids can be either structured (hexahedral) or unstructured (tetrahedral) mesh types depending on the type of discretization scheme; the finite volume method (FVM) was used in this study because of its robustness and unconditional stability ${ }^{11}$ and the nature of geometric configuration used. Concerning the mesh sensitivity analysis, the test performed consisted of increasing the mesh density of the geometrical model in order to properly capture the boundary layer associated problem. Three grid refinement levels are carried out in this study, from coarse to finer mesh. This is done by varying the mesh density to ensure that the CFD solution does not change with further mesh refinement as the aim of grid refinement is basically for numerical accuracy that leads to a solution closer to the exact solution of a system of equations that implies considerable physical approximations. For a $3.5-\mathrm{mm}$ base size used in this study, results obtained for the last two finer meshes are almost identical (see Fig. 2a); hence, it can be established that simulations have reached an asymptotic solution, and the geometry with the last grid refinement level of finer mesh density 2.0 is used to build the fixed bed model. The chosen geometry has 319057 grid cells, 1701035 faces, and 1227886 vertices; from experience, this should be adequate to capture heat transfer phenomena within the porous region. Figure $2 b$ depicts the mesh generated for the experimental model. In this case, a surface remesher was initially applied on the geometry to retriangulate an existing surface in order to improve the overall quality of the surface and optimize it for the volume mesh models. A polyhedral mesh in conjunction with a prism layer mesh model was used to generate orthogonal prismatic cells next to wall boundaries; this is indicated in Fig. 2b. This layer of cells is necessary to allow the solver to resolve near wall flow accurately. This is critical in determining not only the forces and heat transfer on walls but also flow plus features such as separation. Other details on the geometry and mesh design are shown in Table II.

\section{III.B. CFD Modeling}

Modeling is the mathematical problem formulation of the physics involved in terms of a continuous initial
TABLE II

Geometric and Mesh Modeling Parameters

\begin{tabular}{|l|r|}
\hline \multicolumn{1}{|c|}{ Parameter } & Value \\
\hline Bed diameter $(\mathrm{mm})$ & 72 \\
Particle diameter $(\mathrm{mm})$ & 17.46 \\
Cylinder height $(\mathrm{mm})$ & 760 \\
Cylinder wall thickness $(\mathrm{mm})$ & 38 \\
Thermal conductivity, $K_{p}(\mathrm{~W} / \mathrm{m} \cdot \mathrm{K})$ & 15.6 \\
Porous region height $(\mathrm{mm})$ & 500 \\
Fluid region height $(\mathrm{each})(\mathrm{mm})$ & 100 \\
Porosity, $\varepsilon$ & 0.42 \\
Grid base size $(\mathrm{mm})$ & 3.50 \\
Number of grid cells & 319057 \\
Number of faces & 1701035 \\
Number of vertices & 1227886 \\
\hline
\end{tabular}

boundary value problem. In performing any CFD modeling, the physics should first be set up for the model. Essentially, the physics models define the primary variables of the simulation and what mathematical formulation will be used to generate the solution. In the physics model for this study,

1. The fluid was a gas.

2. The gas was assumed to behave as an ideal gas, and the ideal gas law was used for the density temperature dependency.

3. Sutherland's law ${ }^{10}$ was used for the thermal conductivity and viscosity temperature dependencies.

4. The flow was steady and laminar.

5. The space model selected was 3-D because the mesh was generated in three dimensions.

6. The gas specified was helium; its property values are available in the STAR-CCM+ database.

7. As the medium is under natural convection, a coupled solver (iterative) was selected to control the solution due to its robustness for solving flow with dominant source terms, such as buoyancy.

The same experimental boundary conditions (given in Secs. III.D and III.E) were applied to boundaries at the walls and interface in the model. A no-slip shear stress specification was applied at the walls.

\section{III.B.1. Model Analysis}

The 3-D geometry model in Fig. $1 \mathrm{~b}$ consists of an upstream region, a porous region, a downstream region, and two interfaces between the fluid and the porous regions. The upstream and downstream regions 
representing the lower and upper parts of the setup are defined as fluid regions while the region containing the particles is defined as the porous region. An in-place interface is required in between two adjacent regions of different property types; this is to allow for appropriate transfer of quantities of mass and energy calculated during the simulation. The thermal specification for the heated plate located at the bottom of the enclosure is set to a constant heat source corresponding to the experimental value. The external surface of the bottom wall $(Y=0)$ and walls of other regions are considered to be adiabatic (this is only for experimental purposes) while the internal surfaces of the solid walls are assumed to be impermeable to mass transfer. The porous region considered here is homogeneous and isotropic, saturated with helium fluid, and the flow is steady and laminar. Reference values for the fluid thermophysical properties remain constant at initial conditions except density in the buoyancy term, which varies linearly with both temperature and concentration on the assumption that the Boussinesq approximation is implicitly valid. Viscous dissipation in the medium is assumed to be negligible.

Energy transport across the porous region is dealt with by specifying the thermal properties of the region in addition to the thermal properties of the fluid passing through it. Computation of flow is achieved by mathematical models based on conservation principles, namely, the conservation of mass, momentum, and energy governed by the 3-D Navier-Stokes equations representing the fluid as a continuum for an arbitrary control volume. Natural convection is generated by the density differences induced by the temperature differences within a fluid system. By using the physical (interstitial) formulation and assuming a general scalar $\varnothing$, the governing equation in an isotropic porous region expressed in differential form ${ }^{12}$ is shown in Eq. (1) (see the Nomenclature on p. 388):

$$
\frac{\partial(\varepsilon \rho \varnothing)}{\partial t}+\nabla .(\varepsilon \rho \vec{v} \varnothing)=\nabla .(\varepsilon \Gamma \nabla \varnothing)+\varepsilon S_{\varnothing} .
$$

Assuming isotropic porosity and single-phase flow, the volume-averaged mass and momentum conservation equations are given by Eqs. (2) and (3):

$$
\frac{\partial(\varepsilon \rho)}{\partial t}+\nabla \cdot(\varepsilon \rho \vec{v})=0
$$

and

$$
\begin{gathered}
\frac{\partial(\varepsilon \rho \vec{v})}{\partial t}+\nabla .(\varepsilon \rho \vec{v} \vec{v})=-\varepsilon \nabla p+\nabla \cdot(\varepsilon \vec{\tau})+\varepsilon \overrightarrow{B_{f}} \\
-\left(\frac{\varepsilon^{2} \mu}{K} \vec{v}+\frac{\varepsilon^{3} C_{2}}{2} \rho|\vec{v}| \vec{v}\right)
\end{gathered}
$$

The CFD code solves the full set of discretized Navier-Stokes equations using a finite volume approach as earlier mentioned. In Eq. (3), $\partial(\varepsilon \rho \vec{v}) / \partial t$ is the time derivate term, where $\varepsilon$ is the porosity, $\rho$ is the density, and $\vec{v}$ is the velocity vector. The last term in Eq. (3) represents the viscous and inertial drag forces imposed by the pore walls on the fluid; in the last term, $\varepsilon$ is the porosity, $\mu$ is the dynamic viscosity, $K$ is the permeability, $\rho$ is the density, $\vec{v}$ is the velocity vector, and $C_{2}$ is the inertial resistance factor. Porous resistance properties can be specified in several different ways depending on the particular media being modeled and the expected flow conditions through the media. Two methods are widely used when specifying porous resistance, namely, the orthotropic viscous resistance method and the isotropic resistance method. In this simulation, the choice of isotropic resistance using the Ergun equation was made.

In the porous region, the theoretical pressure drop per unit length was determined using the Forchheimer equation. ${ }^{13}$ This is represented in Eq. (4):

$$
-\frac{\Delta P}{L}=\frac{\mu}{k_{\mathrm{p}}} u_{o}+\beta \rho u_{o}^{2}=\left(P_{\nu} u_{o}+P_{i} u_{o}^{2}\right) .
$$

An example of the above Forcheimer equation widely used in porous media flow is the Ergun equation, ${ }^{14}$ a semiempirical correlation applicable over a wide range of Reynolds number and for many types of packing:

$$
-\frac{\Delta p}{L}=\frac{150 \mu(1-\varepsilon)^{2} u_{o}}{\varepsilon^{3} d_{p}^{2}}+\frac{1.75 \rho(1-\varepsilon) u_{o}^{2}}{\varepsilon^{3} d_{p}},
$$

where $u_{o}$ is the superficial velocity through the porous region and $P_{i}$ and $P_{v}$ are coefficients defining the porous resistance, known as the inertial resistance and viscous resistance, respectively. Values for the resistance coefficients can be measured experimentally or derived using various empirical relationships, depending on the exact nature of the problem. When modeling laminar flow through a packed bed, the second term in Eq. (5) is usually dropped resulting in the Blake-Kozeny equation. ${ }^{14}$

\section{III.B.2. Wall and Interfacial Heat Transfer Coefficient in the CFD Model}

For viscous flows as experienced in this model, the central concept for modeling the convective heat transfer coefficient evaluated at the computed wall temperature $T_{w}$ when heat is being transferred to the wall boundary at specified local heat flux $q_{w}$ between fluid and solid phases in the region is expressed by Eq. (6). 
In obtaining the heat transfer coefficient at the porous region wall boundary $h_{w}$, it is necessary that an associated fluid reference temperature be specified at the heat transfer coefficient field function:

$$
h_{w}=\frac{q_{w}}{\left|\left(T_{f}-T_{w}\right)\right|} \text {. }
$$

Considering an interfacial heat transfer area $A_{i}$ between the fluid and the porous region, a specific surface area $a_{i}=A_{i} / \Delta V$, which is the solid surface area per unit volume of the solid, is used; the interfacial convective heat transfer coefficient $h_{i}$ is expressed by Eq. (7):

$$
\begin{gathered}
h_{i} a_{i}\left(\left(T_{s}\right)^{i}-\left(T_{f}\right)^{i}\right)=\frac{1}{\Delta V} \int_{A_{i}} \mathbf{n}_{i} \cdot k_{f} \nabla T_{f} d A \\
=\frac{1}{\Delta V} \int_{A_{i}} \mathbf{n}_{i} \cdot k_{s} \nabla T_{s} d A,
\end{gathered}
$$

where

$$
\begin{aligned}
\left(T_{s}\right)^{i},\left(T_{f}\right)^{i}= & \text { intrinsically average temperature of } \\
& \text { solid and fluid phases, respectively } \\
k_{f}, k_{s}= & \text { thermal conductivity of fluid and solid } \\
& \text { phases, respectively } \\
\mathbf{n}_{i}= & \begin{array}{l}
\text { unit vector normal to the fluid-porous } \\
\text { interface, pointing from the fluid } \\
\text { toward the porous region. }
\end{array}
\end{aligned}
$$

The Nusselt number based on the fluid temperature $T_{f}$ is a useful quantity that can be used for characterizing the heat transfer phenomenon. The Nusselt number, expressed by Eq. (8), is based on hydraulic diameter $d_{h}$ and the effective fluid conductivity using Eq. (6):

$$
\mathrm{Nu}_{w}=\frac{q_{w} d_{h}}{k_{e f f}\left(T_{f}-T_{w}\right)} .
$$

The Rayleigh number, expressed by Eq. (9), is a function of the Grashof and Prandtl numbers; this is another important dimensionless parameter used in evaluating the heat transfer performance of a porous medium:

$$
\mathrm{Ra}=\frac{g \beta\left|T_{w}-T_{f}\right| d_{h}^{3}}{v^{2}} \operatorname{Pr} .
$$

\section{III.C. Discretization Scheme}

In the CFD analysis, each type of discretization method (FVM, finite difference method, and finite element method) used should yield the same solution if the grid is fine enough, but some methods are more suitable to some cases than others. In the selection of discretization methods, efficiency, accuracy, and special requirements (i.e, shock wave tracking) are some factors that should be considered. Higher-order numerical methods usually predict a higher order of accuracy for CFD but are more likely unstable due to less numerical dissipation. Stability is attained if the method adopted does not magnify the errors that appear in the course of the numerical solution process. A finite volume discretization method is used in this work. The solution domain is subdivided into a finite number of small control volumes corresponding to the cells of a computational grid. Discrete versions of the integral form of the continuum transport equations are applied to each control volume. The objective is to obtain a set of linear algebraic equations, with the total number of unknowns in each equation system corresponding to the number of cells in the grid. With the nonlinear equation, iterative techniques that rely on suitable linearization strategies must be employed. The resulting linear equations are then solved with an algebraic multigrid solver. Considering the general governing equation given in Eq. (1), which consists of the transient term, convective term, diffusion term, and source term, respectively, the discretization approach for the individual term differs. The transient term is only included in transient calculations; it is not generally used as a device to obtain a steady-state solution. The Implicit Unsteady solver in STAR-CCM + offers two temporal discretization options: first order and second order. The first-order temporal scheme, also referred to as Euler Implicit, discretizes the unsteady term using the solution at the current time level $n+1$ as well as the one from the previous time level $n$. This is shown in Eq. (10):

$$
\frac{d}{d t}(\rho \chi \varnothing V)_{0}=\frac{\left(\rho_{0} \varnothing_{0}\right)^{n+1}-\left(\rho_{0} \varnothing_{0}\right)^{n}}{\Delta t} V_{0} .
$$

The second-order temporal scheme expressed by Eq. (11) discretizes the unsteady term using the solution at the current time level, $n+1$, as well as those from the previous two time levels, $n-1$ :

$$
\frac{d}{d t}(\rho \chi \varnothing V)_{0}=\frac{3\left(\rho_{0} \varnothing_{0}\right)^{n+1}-4\left(\rho_{0} \varnothing_{0}\right)^{n}+\left(\rho_{0} \varnothing_{0}\right)^{n-1}}{2 \Delta t} V_{0} .
$$

On the first time step of a second-order temporal simulation, a first-order discretization is used since only two time levels are available. The convective term at a face is discretized using the expression in Eq. (12): 


$$
[\varnothing \rho(\mathrm{v} \cdot \boldsymbol{a}-\boldsymbol{G})]_{f}=(\dot{m} \varnothing)_{f}=\dot{m}_{f} \varnothing_{f},
$$

where

$$
\begin{aligned}
\varnothing_{f}, \dot{m}_{f}= & \text { scalar values and mass flow rates at the } \\
& \text { face, respectively } \\
\mathbf{G}= & \begin{array}{l}
\text { grid flux computed from the mesh } \\
\text { motion. }
\end{array}
\end{aligned}
$$

The manner in which the face value $\varnothing_{f}$ is computed from the cell values has a profound effect on the stability and accuracy of the numerical scheme. Several schemes are commonly used, some of which include first-order upwind, second-order upwind, central differencing, bounded central differencing, blended upwind/central, etc.

\section{III.D. Initial State and Boundary Conditions}

The initial state conditions for the two models considered in this study are the same. The working fluid is specified as helium gas at initial static temperature $293.1 \mathrm{~K}$, static gauge pressure $0.0 \mathrm{~Pa}$, and reference pressure $86.6 \mathrm{kPa}$ (value of $1 \mathrm{~atm}$ as obtainable in this part of the world). The boundary conditions in the first model are the same as in the experimental setup: The cylinder wall boundary is specified as adiabatic and at no-slip wall conditions. At the upstream fluid region, a constant heat source thermal specification of $750 \mathrm{~W}$ is applied to the bottom heated plate. Static temperatures of 460 and $400 \mathrm{~K}$ are specified for the upstream and downstream porous in-place internal interface boundaries, respectively. The velocity inlet and pressure outlet at default values of $1 \mathrm{~m} / \mathrm{s}$ and $0.0 \mathrm{~Pa}$, respectively, are specified for both internal interface boundaries. No thermal energy source is specified at the downstream fluid region. A heat source thermal specification of $-300 \mathrm{~W}$ for heat extraction by the bed particles in the porous region is applied at the cylindrical curved wall boundary face. In the second model, the cylinder wall boundary is specified as convection due to the expected heat transfer across the cladding wall. The boundary type for both the downstream and upstream in-place internal interfaces is specified as pressure outlet boundary condition of $460 \mathrm{~K}$ static temperature and 0.0-Pa gauge pressure, respectively. It is worth noting that initial conditions do not affect final results but rather affect the convergence path (number of iterations or time steps), and a converged solution is one that is nearly independent of meshing errors.

\section{III.E. Simulation Method}

On completion of the geometry, meshing, and physics model processes, porosity and porous resistance property values need to be specified in characterizing the medium. Porous resistance coefficients are represented by inertial and viscous terms. These coefficients are required for the source terms in the momentum equation as they affect the fluid flow through a region in a particular direction. Two models were simulated. The material properties of the bed particles and the cylinders in both models are the same. The wall boundary conditions and heat source generation in both models differ. The first model is a mimic of the experimental investigation while the second model is patterned after the cladding tube of the new fuel design.

In the simulation of the first model (see Fig. 3a), no porous inertia resistance was active; only porous viscous resistance was active because the flow is assumed to be laminar. Simulating a porous region under natural convection requires specifying the gravity $(-9.81 \mathrm{~m} / \mathrm{s})$ in the right direction in accordance with the model coordinates and also the fluid properties (i.e, density); this prepares the medium for buoyancy-driven effects to be simulated. Energy transport across the porous region is dealt with by specifying the thermal properties of the solid represented by the porous media in addition to the thermal properties of the fluid passing through it. Specified values are given in Table I.

In the simulation of the second model (see Fig. 3b), a constant heat source of $750 \mathrm{~W}$ is specified at the porous region because the particles are a heat-generating source like the coated fuel particles. The simulation in this model is carried out at a steady state because the heat transfer within the bed is relatively uniform. For the present study in the experimental setup of 410 uniformly sized particles randomly packed in a container, the porous region in the simulation is characterized by the following parameters:

1. porosity: 0.42

2. packing factor: 0.58

3. helium gas density: $0.16648 \mathrm{~kg} / \mathrm{m}^{3}$

4. solid (particle) density: $7913 \mathrm{~kg} / \mathrm{m}^{3}$

5. solid (particle) specific heat: $456 \mathrm{~W} / \mathrm{kg} \mathrm{K}$

6. solid (particle) thermal conductivity: $15.6 \mathrm{~W} / \mathrm{m} \mathrm{K}$.

A couple solver is selected to control the solution of continuity, momentum, and energy equations in vector form and is activated once per iteration (or once per time step for explicit unsteady simulations). Appropriate numerical parameters (convergence limit, underrelaxation factor, number of iterations/time steps, etc.) are specified to control the calculation. The simulation is run after initialization, and the simulation time ranged from 1 to 36 $\mathrm{h}$ depending on the studied case; the solution diverges for the first few numbers of iterations. Numerical convergence of the model was checked based on a suitable diminution of the normalized numerical residuals of 


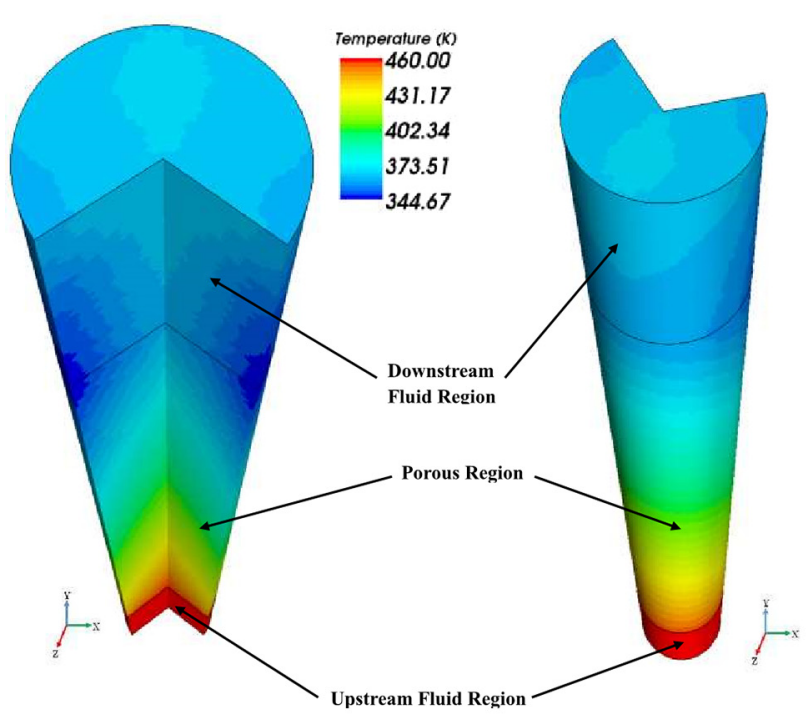

(a)

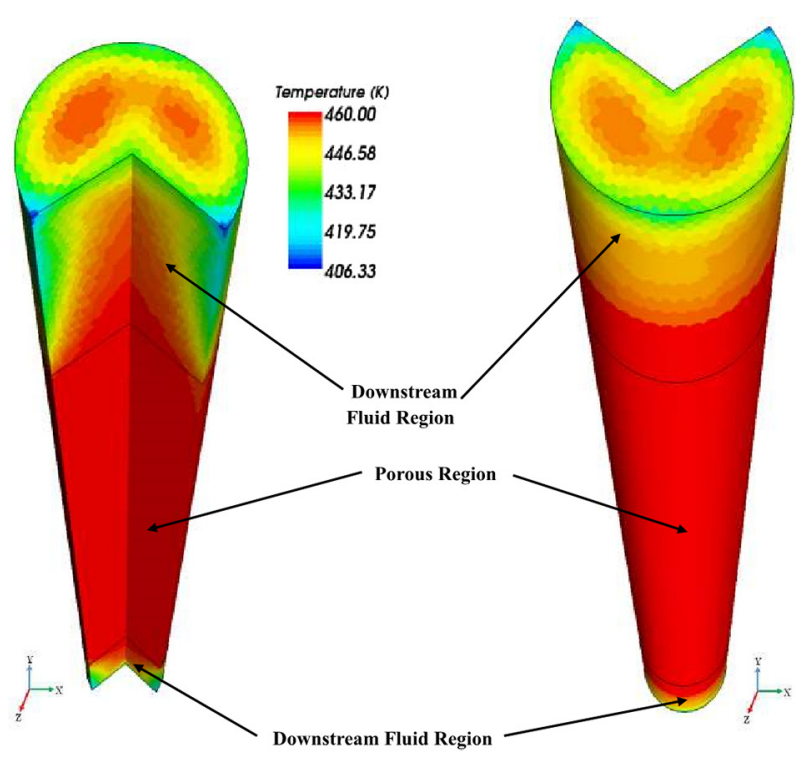

(b)

Fig. 3. (a) Temperature contour plot across fluid and porous regions in heated bottom plate geometrical model. (b) Temperature contour plot across fluid and porous regions in particle generating heat source geometrical model.

computed variables. Residual monitor plots of conserved variables such as mass, momentum, and energy against the iteration number are very useful for judging the convergence (or divergence) of a solution, and they are created automatically within every simulation. However, it is important to understand both the significance of residuals and their limitations. While it is true that the residual quantity will tend toward a very small number when the solution is converged, the residual monitors cannot be relied on as the only measure of convergence. Other convergence criteria used in this study are creating scalar and vector plots of the entire solution domain or parts of it, and visualizing these plots as the solution develops, this technique is useful when comparing to residuals that cannot exhibit a converged solution. $X-Y$ plots of temperature, heat transfer coefficient, Rayleigh number, and Nusselt number of the results at the porous region wall boundary are reported in the graphs of Figs. 4, 5, and 6.

\section{VALIDATION}

The following guidelines are crucial to validate the experimental results for the convective fluid-to-particle heat transfer characteristics with CFD simulation:

1. developing a geometrical model of the prototype either to the same size or scaled

2. applying the same initial and boundary conditions used under the experimental condition
3. using the same thermal specifications as applicable during the experiment

4. following the right simulation process or methodology.

In the course of this study, an experimental investigation was carried out to evaluate the natural convection heat transfer characteristics in a porous medium heated from below. A basic cell unit microscopic approach

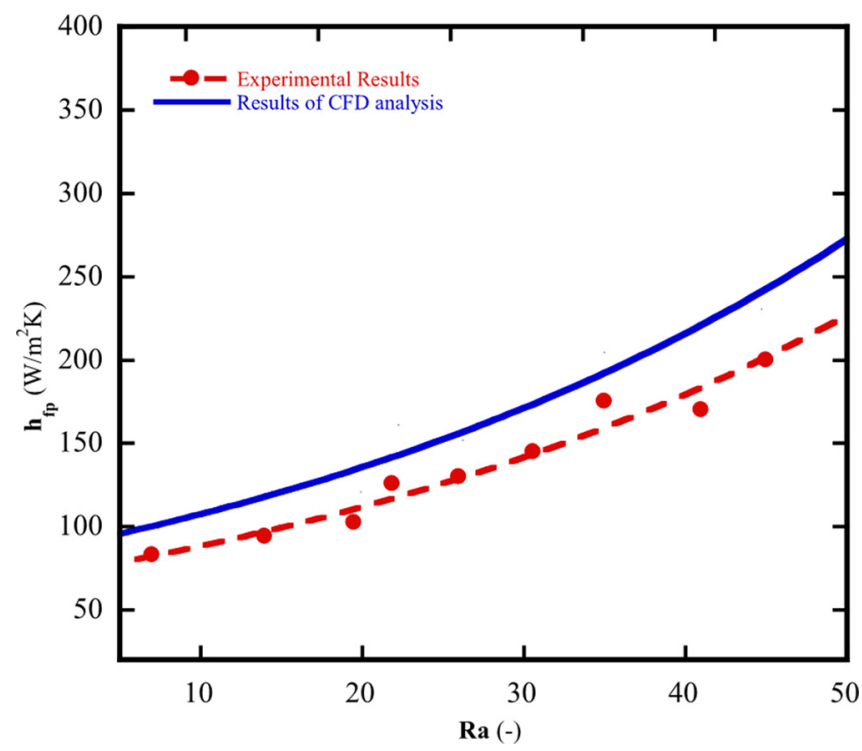

Fig. 4. Variation of convective fluid-to-particle heat transfer coefficient with Rayleigh number for measured and simulated results in a porous region heated from below $(N=4.124)$. 


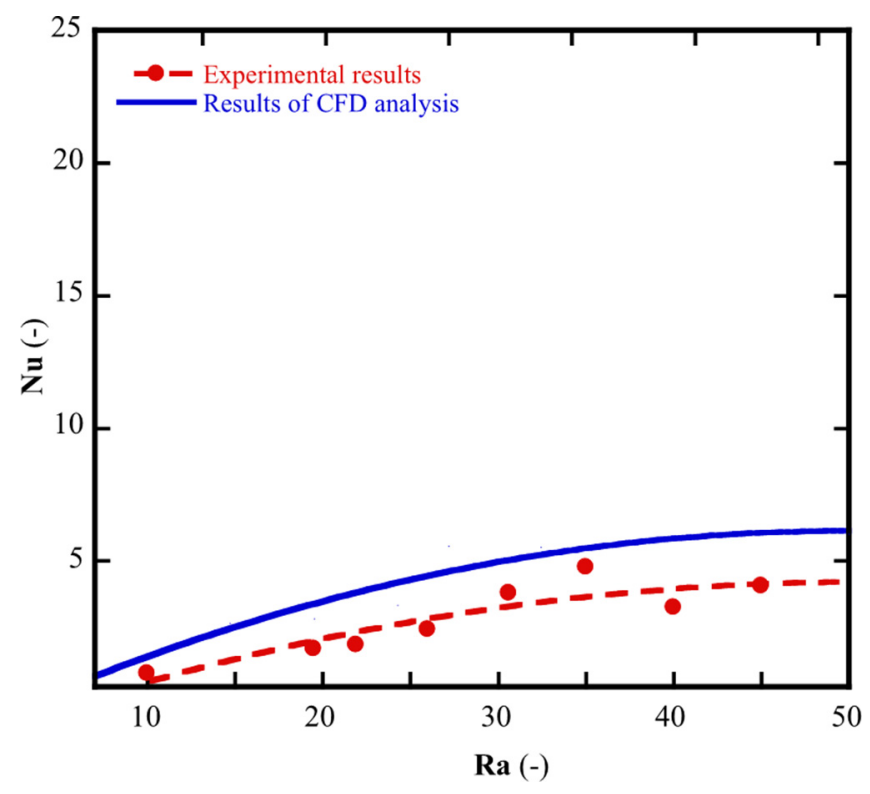

Fig. 5. Impact of Rayleigh number on convective fluidto-particle Nusselt number for measured and simulated results at the porous region heated from below $(N=$ 4.124).

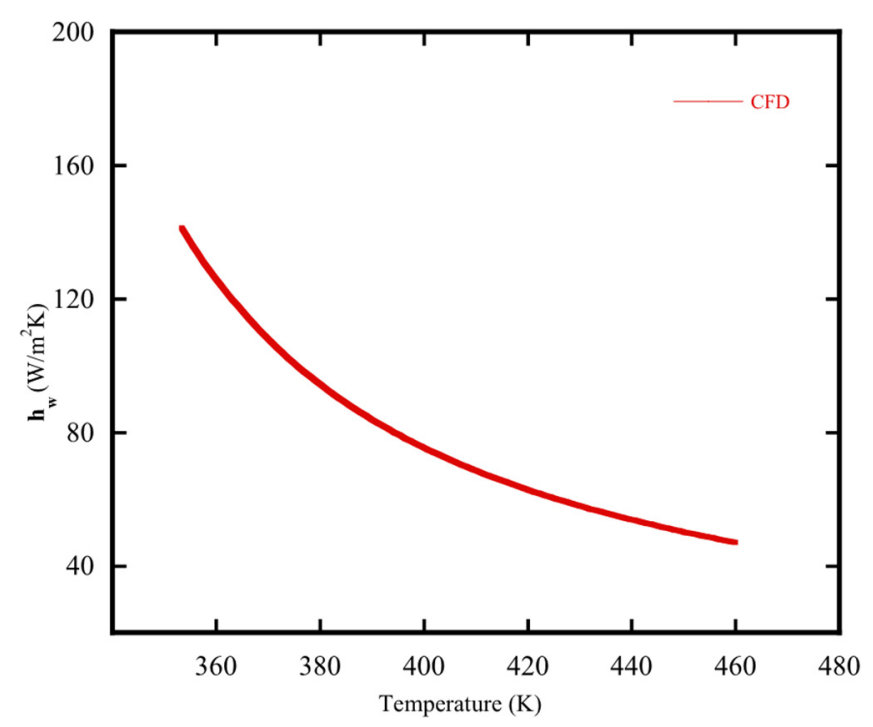

Fig. 6. Effect of temperature on particle-to-fluid heat transfer coefficient at the simulated porous region.

requiring a conservative thermal energy balance was adopted in analyzing the heat transfer characteristics in the medium considered. Results of the validation displayed graphically in Figs. 4 and 7 reveal some differences through the gap between the experimental and numerical results while the trends are the same. A number of reasons may be responsible for the differences, which include uncertainty from simulation error and has been the noticeable difference between the simulation and experimental results. It is assumed to comprise additive

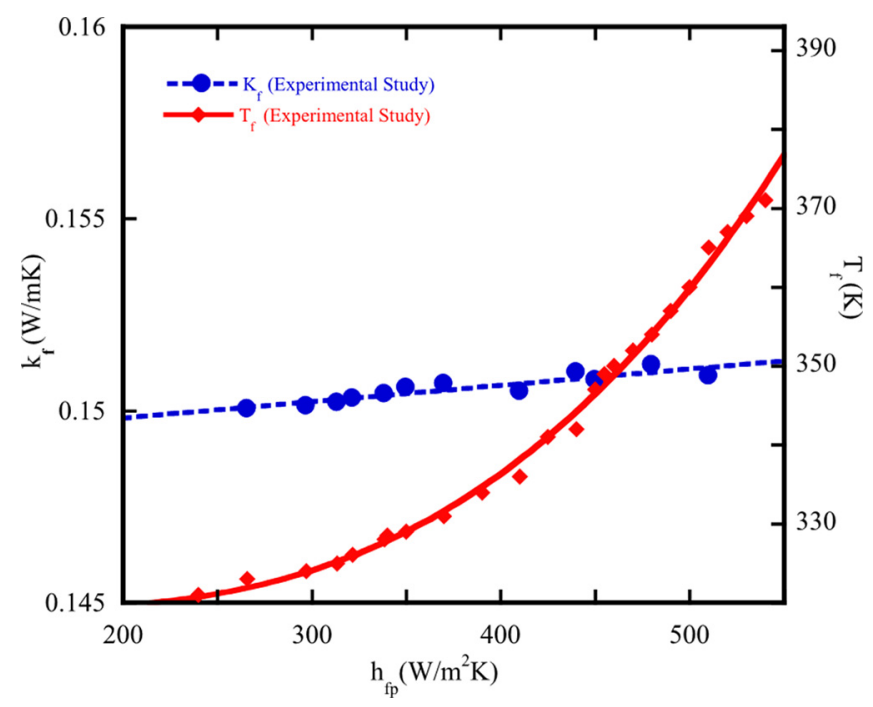

Fig. 7. Effect of gas thermal conductivity on convective fluid-to-particle heat transfer coefficient in an increasing packed bed temperature.

modeling and numerical errors. Another reason could be inaccurate thermomechanical medium properties at the input stage as this will not allow for proper accounting of thermal expansion during the simulation exercise.

\section{IV.A. Porous Media Heated from Below}

The experimental setup in Figs. 1a and 1c is a porous medium supplied with heat energy at a steady rate through a heated bottom steel plate; the medium exhibits a local thermal nonequilibrium, negligible thermal dispersion effects, and an adiabatic cylindrical wall. Fluid flow and heat transfer in the medium is one dimensional; bed particles and the entire packed beds exhibit one-dimensional transient heat conduction as shown in Figs. 8a and 8b. The heat transfer coefficient $h_{f p}$ (fluid to particle) in the medium obtained from the thermal energy balance ${ }^{15}$ is expressed in Eq. (13):

$$
\begin{gathered}
h_{f p}= \\
(1-\varepsilon) \frac{\left(\rho C_{p} V_{p}\right)}{\Delta t}\left[T^{t+\Delta t}-T^{t}\right] \\
-\sum_{m=1}^{4}\left[\left\{k_{e f f}^{c} \frac{d T_{i}}{d z} A_{c}+\frac{\Delta T_{i}}{R_{G}}\right\}_{B c}+\left\{A_{p} k_{\text {eff }}^{r} \frac{d T_{i}}{d z}\right\}_{B c}\right]_{m} \\
+\sum_{m=1}^{4}\left[\left\{k_{\text {eff }}^{c} \frac{d T_{i}}{d z} A_{c}+\frac{\Delta T_{i}}{R_{G}}\right\}_{T c}+\left\{A_{p} k_{e f f}^{r} \frac{d T_{i}}{d z}\right\}_{T c}\right]_{n} \\
\left(A_{p}-\sum_{e=1}^{8} A_{c_{e}}\right)\left(T_{f}-T_{p}\right)
\end{gathered}
$$




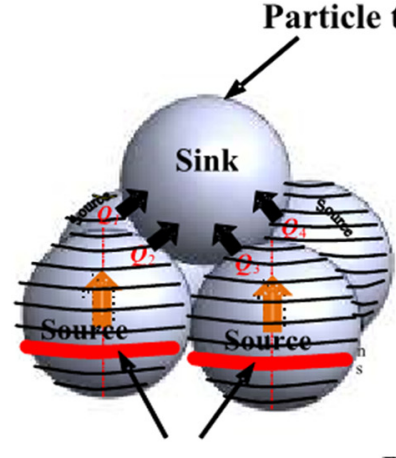

Control volumes

(a)

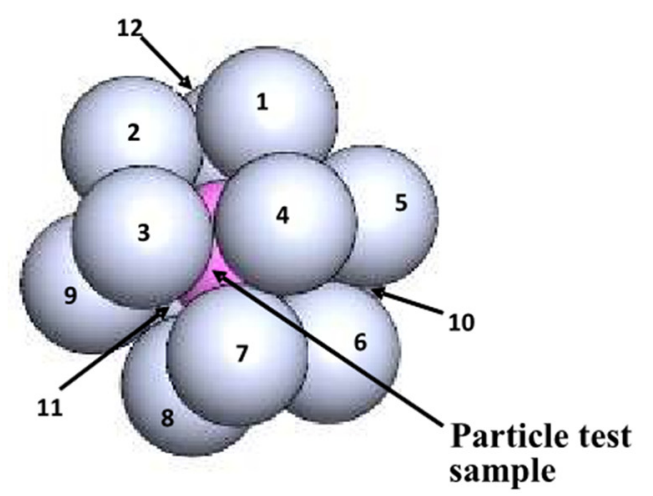

(c)

Fig. 8. (a) Contacting particles at the lower half of particle test sample. (b) Contacting particles at the upper half of particle test sample. (c) Maximum possible number of contacting particles for structured packing. ${ }^{31}$

The first term of the numerator on the right side of Eq. (13) is the heat stored within the particle test sample. The second term represents the heat supplied to the particle test sample by adjacent contacting test particles (depending on the random placement of particles, usually is in the range of one to four) below it; this serves as a heat source while the particle test sample serves as a heat sink. The last term represents the heat supplied by the particle test sample to adjacent contact particles (usually in the range of one to four) above it. The heat transferred to and from the particle test sample in both instances is by conduction and radiation at the contact points as expressed by Eq. (13). The surface area of the particle test sample exposed to convective heat transfer is the difference between the spherical particle total surface area $A_{p}$ and the sum of $e$ contact area spot located on the surface of the particle test sample. Because the particle test sample and adjacent contact test particles are not in isolation but are in a porous medium, the solid volume fraction $(1-\varepsilon)$ of the solid phase in Eq. (13) is applied.
Equation (13) is a combination of both analytical models (concepts of the thermal contact resistance of smooth sphere particles) and numerical models (FVM used in determining the temperature distribution of cells located within the particle test sample). $R_{G}$ is the resistance of interstitial gas in the macrogap considering a Hertzian contact $\left(H_{B}<1.3 \mathrm{GPa}\right)$ (Ref. 16) between adjacent particles in the experimental setup. The macrogap is located within the contact region between adjacent particles, and this gap accounts for a large percentage of the heat transferred by conduction between contacting particles.

\section{IV.B. Porous Media Heated by Bed Particles}

Practical applications of heat generation from bed fuel elements to the working fluid could be found in HTRs fueled with spherical fuel and also in thermal energy storage systems functioning as a recuperator. Despite over 50 years of theoretical and experimental research, such as Refs. 17 through 23, there has been no consensus concerning the correlations to use to predict effective heat transfer characteristics in fixed packed beds. If accuracy is to be given preference over simplicity, then a theoretical and analytical solution of the conservation of energy equation in the medium is the best approach because it accounts for the thermophysical properties of contacting sphere particles, the interstitial gas effect, gas temperature and pressure, and diameter of spheres.

Figure 9 is a schematic diagram of the proposed fuel design containing fuel in the form of loose coated particles in a helium environment placed inside the nuclear fuel cladding tube of the fuel assemblies. Each particle fuel in the tube generates heat by nuclear fission; heat transfer by a particle fuel in the proposed design is in both axial and radial directions. The gas coolant absorbs the heat generated, which in turn heats the surrounding water.

Considering a basic unit cell in the medium, Eq. (14) is particle-to-fluid heat transfer coefficient $h_{p f}$ obtained from the conservative thermal energy balance for particleto-fluid heat transfer in the medium of the proposed $\operatorname{design}^{15}$ :

$$
\begin{gathered}
Q_{n u}-(1-\varepsilon) \frac{\left(\rho c_{p} V_{p}\right) \Delta T}{\Delta t} \\
h_{p f}=\frac{-\left\{\bar{N}_{c}\left[k_{e f f}^{c} \frac{d T}{d z} A_{c}+\frac{\Delta T}{R_{G}}\right]-A_{p} q_{R}^{\prime \prime}\right\}}{\left(A_{p}-\bar{N}_{c} A_{c}\right)\left(T_{p}-T_{f}\right)},
\end{gathered}
$$

where

$$
\begin{aligned}
Q_{n u}= & \text { heat generated by nuclear fission in the } \\
& \text { coated particle fuel }
\end{aligned}
$$




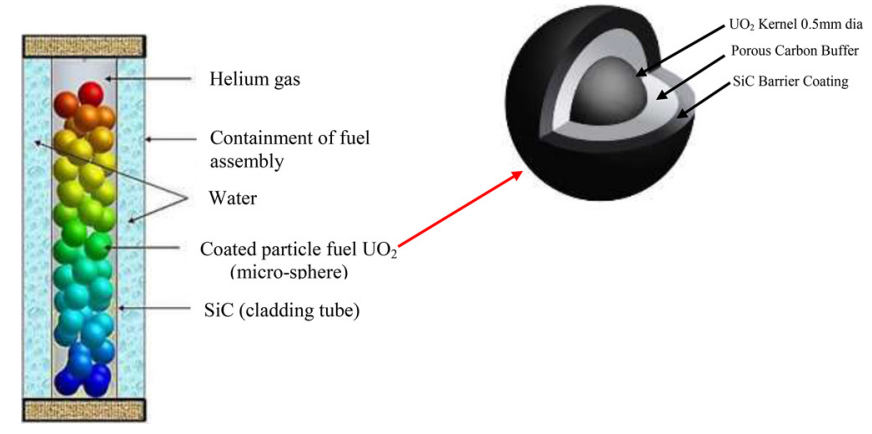

Fig. 9. Schematic of proposed coated particle fuel design in a $\mathrm{SiC}$ cladding tube.

$$
\begin{aligned}
N_{c}= & \text { average coordination number or average } \\
& \text { kissing number, }{ }^{24} \text { which is a function of both } \\
& \text { the bed porosity and particle diameter. }
\end{aligned}
$$

The heat transfer performance in the proposed new fuel design under natural convection can also be evaluated using the heat transfer coefficient $h_{p f}$, Nusselt number $\mathrm{Nu}$, Grashof number Gr, Prandtl number Pr, and Rayleigh number Ra. These are expressed in Eqs. (15) and (16):

$$
\mathrm{Nu}_{d_{p}}=\frac{h_{p p} L_{c}}{k_{f}}
$$

and

$$
\mathrm{Ra}_{d_{p}}=\operatorname{Gr}_{d_{p}} \operatorname{Pr}=\frac{g \beta\left|T_{p}-T_{f}\right| L_{c}^{3}}{v^{2}} \operatorname{Pr} .
$$

\section{IV.C. Thermal and Irradiation Induced Creep Effects in the Cladding}

The proposed fuel is to be designed in the same pattern as the TRISO particle fuels (see Fig. 9) embedded in a graphite matrix, which greatly limit the release of fission products even at temperatures $>1600^{\circ} \mathrm{C}$ (Refs. 25 and 26). The structural integrity of the graphite components in the reactor core, along with the contributions of temperature, irradiation, and load, has a strong impact on the entire reactor's safety. ${ }^{27,28}$ Creep exists when nuclear graphite under a load is exposed to high temperature and fast neutron irradiation conditions. Basically, there are two components of creep in graphite, thermal and irradiation induced, with the latter dominating as carbon atoms are constantly displaced under a barrage of high-energy neutrons. This leads to "damage" of the structure at the atomic level, and this is manifest in large components by a dimensional change (first in the form of shrinkage and later in the form of expansion), bowing, changes in strength parameters such as Young's modulus, in thermal properties, and in potential cracking. Creep under continuing irradiation serves to alleviate the stresses and to delay the onset of damaging cracks.

In relation to the coated fuel particles, the changing effect of the coefficient of thermal expansion due to high temperature and irradiation has a major impact on the design of graphite components. To avoid excessively high thermal strain and stress caused by temperature changes, the thermal expansion behavior and related factors need to be researched.

\section{IV.D. Proposed Nuclear Fuel Design Safety Enhancement}

Accidents resulting in the release of fission products are principally driven by the degradation of the barrier due to excessive temperature. Reactors are normally shut down due to rising fuel temperature, but the radioactive fission product keeps on generating heat even after shutdown. This residual heat is called decay heat. In "conventional" reactors, heat is removed by active cooling systems (such as pumps), which rely on the presence of coolant such as water. Any such system may fail, and therefore, they are duplicated in conventional reactors to make sure that there will be support, should the first line of defense fail.

The proposed nuclear fuel design in the cladding (see Fig. 4) is tailored toward the concept of HTR nuclear fuel pebbles. Particles of enriched uranium dioxide are coated by three layers: pyrolytic carbon, silicon carbide, and pyrolytic carbon (to make them safer). These three layers are to protect the fuel itself in case of a complete loss of cooling. ${ }^{29}$ The safety advantage of this fuel design is that it utilizes the concepts of passive safety compared to the active safety system utilized by the traditional fuel (see Fig. 4b). Adherence to the following three well-known principles is necessary to achieve these enhanced safety measures $^{30}$ :

1. Control the heat production in the fuel by limiting decay heat levels and excessive reactivity excursions.

2. Ensure sufficient heat removal capability (including the options of doing this by passive means, so that the fuel will not exceed the specified limit).

3. Limit the possibilities of chemical degradation of the fuel due to large-scale air or water ingress (in this case, the problem is that the $\mathrm{SiC}$ coating can be destroyed by corrosion when temperatures exceed $\left.1200^{\circ} \mathrm{C}\right)$. 


\section{RESULTS AND DISCUSSION}

On completion of the modeling exercise, simulation results were compared with analyzed experimental results obtained from the investigated porous medium heated from below. Discrepancies between the two results were noticed, and a major reason for these discrepancies could be errors and uncertainties resulting from both approaches. In general, an uncertainty implies a parameter associated with the result of a measurement, or the combined result of several measurements, that characterizes the dispersion or the width of a specific confidence interval. There are three different types of uncertainties that are considered to contribute to the total uncertainty in the test facility: statistical variance, instrument uncertainty, and drift uncertainty. Statistical variance $u\left(x_{i, \text { statistical }}\right)$ is obtained using the normal variance expression. The instrument uncertainty $u\left(x_{i, i n s t r u m e n t}\right)$, on the other hand, is obtained from the manufacturer, and the drift uncertainty $u\left(x_{i, d r i f t}\right)$ is obtained after each major calibration. The uncertainties are then combined to give a total standard uncertainty expressed by Eq. (17):

$$
\begin{gathered}
u\left(x_{i, \exp }\right)= \\
\sqrt{\left[u\left(x_{i, \text { statistical }}\right)\right]^{2}+\left[u\left(x_{i, \text { instrument }}\right)\right]^{2}+\left[u\left(x_{i, \text { drf } t}\right)\right]^{2}} .
\end{gathered}
$$

Calculation results revealed that the uncertainty is found to be $16.8 \%$. Errors and uncertainty are unavoidable aspects of CFD modeling. Such errors and uncertainty could emanate from round-off error (error caused by computational representation of real numbers by means of a finite number of significant digits), iterative convergence error (numerical errors resulting from truncating the iteration of discretized governing equations before a final or exact solution is reached), discretization error (error from representing a function by its values at a discrete set of points), input uncertainty (inaccuracies due to limited information or approximate representation of geometry, boundary conditions, fluid/material properties), and physical model uncertainty (discrepancies between real flows and CFD due to inadequate representation of physical/ chemical processes or due to simplifying assumptions in the modeling process). Some of these errors and uncertainties are quantified in the study by methods of verification and validation, and appropriate measures are taken to reduce them to the barest minimum during the simulation process as mentioned in the paper. The nonelimination of these errors and uncertainty in both approaches gave rise to discrepancies between the experimental and simulation results.
Figure 3a depicts temperature contours for the simulated packed beds. It demonstrates how the temperature field changes across the regions in the medium under buoyancy-driven flow within the cylindrical enclosure for a given relatively steady heat source supplied by an inductively heated steel plate located at the bottom of the cylinder. The heat conduction in this medium through the color bar indicator is observed to be transient. It can be seen that the upstream fluid region in the red contour is relatively uniform but temperature changes begin to manifest as we move away from this region into the porous region and to the downstream region. On the lower part of the porous region, the red/yellowish contour shows the influence of the hot helium gas on particles located at the lower part of the porous region as they extract most of the heat from the hot gas leaving the upstream region. The particles at this part of the porous region thus become hotter than the particles at the upper part of the porous region. This makes the gas denser and unable to flow to the end of the downstream region at the initial stage due to a change in the flowing gas density, but after the accumulation of heat by a convectional current taking place in the medium, the gas gradually flows to the downstream end section, and flow recirculation continues. A similar experience from captured data was observed during the experimental investigation.

Depicted in Fig. 3b are the temperature contours for the heat source-generating packed beds. A simulation result for bed particles generating the heat source with 750 $\mathrm{W}$ at maximum temperature of $460 \mathrm{~K}$ is shown in Fig. 3 b. It can be seen that the particle surface temperatures in the red contour inside the porous region are uniform, and on the side walls of the region, the red contour shows the influence of conduction at each particle-to-wall point contact, and the heat spread out influenced and facilitated by the hot helium gas neighboring the wall boundary along the axial direction. At the upstream and downstream regions, the red/yellow/green contour is influenced by the heat transfer spread out along the tube wall facilitated by the hot gas temperature at these two locations as there are no particles placed in the regions. The red/brownish contour at the top wall of the downstream region indicates the influence of less dense hot helium gas experiencing recirculation concentrated on this part of the top wall boundary. The yellow/green contour at the top wall of the downstream region indicates heat spread out from parts experiencing recirculation on the wall and also the influence of less dense hot gas on the wall boundary. After $\sim 45 \mathrm{~min}$ of simulation run, if the wall boundary is made adiabatic, the medium will reach a state of local thermal equilibrium (LTE) if no heat is extracted from the gas. In the proposed nuclear fuel design, the heat from the gas is 
to be extracted by the surrounding water outside the cladding tube; this prevents the medium from attaining a condition of LTE. The wall boundary in this model is specified as convection to allow heat transfer across the cladding to the surrounding water to prevent the medium from reaching a state of LTE.

Figure 7 depicts the effect of thermal conductivity of helium gas with the heat transfer coefficient in an increasing bed temperature. Figure 4 shows that the fluidto-particle heat transfer coefficient increases with an increasing gas temperature at slightly increasing gas thermal conductivity. Figure 4 compares the measured and simulated results of the variation of the convective fluidto-particle heat transfer coefficient with the Rayleigh number for the porous medium heated from below. Supplied with the constant heat source thermal specification of the same magnitude as obtained in the experiment, it is observed through the curves that a percentage error of $20 \%$ exists between the simulated and experimental results. This can probably be attributed to the fact that the simulated values are computed using the macroscopic approach considering the lumped parameter effect while the measured values are generated using a microscopic basic cell unit approach. Other reasons may be experimental and simulation errors. Despite these discrepancies, the comparisons showed fairly good qualitative similarities between the CFD and the experimental results.

The results in Fig. 5 illustrate the effect of the Nusselt number on the Rayleigh number for a porous medium heated from below. A percentage error of $\sim 35 \%$ occurs between the measured values and the simulated values of $\mathrm{Ra}$ and $\mathrm{Nu}$ and could be due to uncertainties and inaccurate input of the thermomechanical properties of the bed materials. A fair agreement still exists between the two results. The result depicted for a particle heat-generating source porous medium in Fig. 6 illustrates an increasing fluid temperature effect on the particle-to-fluid heat transfer coefficient. It was observed from the simulated results that as the fluid temperature in the medium increases and approaches the wall temperature, the heat transfer coefficient declines toward zero as the medium tends toward a state of LTE. The presence of the surrounding water will prevent the medium from nearing the state of LTE.

\section{CONCLUSION}

This study has demonstrated the use of a porous region modeling approach in validating fluid-to-particle heat transfer characteristics in a porous medium under natural convective conditions. Though the simulation result values for $h_{w}, \mathrm{Nu}_{w}$, and $\mathrm{Ra}$ in the medium investigated have some percentage errors compared with the experimental measured results, a similar profile was observed. It is believed that the discrepancy in the results is a consequence of lumped parameter effects in the porous region modeling approach used although other reasons like uncertainties are not ruled out. If the percentage error between the simulated and experimental results could be reduced to the barest minimum, this approach could give confidence in the application of a porous region modeling approach in predicting convective heat transfer characteristics of fixed beds or packings in general.

It is believed that this approach could be useful in the study of the heat transfer characteristics in a medium containing coated particle fuels if errors due to uncertainties are reduced drastically. This will be a good means to simulate packed beds with a large number of bed particles using a system with an average-sized computational capacity.

\section{Nomenclature}

$$
\begin{aligned}
A & =\text { area }\left(\mathrm{m}^{2}\right) \\
a & =\text { specific surface area }\left(\mathrm{m}^{-1}\right) \\
\mathbf{a} & =\text { face area vector }\left(\mathrm{m}^{2}\right) \\
\vec{B} & =\text { body force }(\mathrm{N}) \\
C_{2} & =\text { inertial resistance factor } \\
c_{p} & =\text { specific heat at constant pressure }(\mathrm{J} / \mathrm{kg} \mathrm{K}) \\
d & =\text { diameter }(\mathrm{m}) \\
g & =\text { gravitational acceleration }\left(\mathrm{m} / \mathrm{s}^{2}\right) \\
h & =\text { heat transfer coefficient }\left(\mathrm{W} / \mathrm{m}^{2} \mathrm{~K}\right) \\
K & =\text { permeability }\left(\mathrm{m}^{2}\right) \\
k & =\text { thermal conductivity }(\mathrm{W} / \mathrm{m} \mathrm{K}) \\
L & =\text { bed height }(\mathrm{m}) \\
L_{c} & =\text { characteristic length }(\mathrm{m}) \\
N & =\text { diameter ratio, } d_{b} / d_{p} \\
N_{c} & =\text { average coordination number } \\
P & =\text { pressure }(\text { Pa) } \\
P_{i} & =\text { inertial resistance coefficient } \\
P_{v} & =\text { viscous resistance coefficient } \\
q^{\prime \prime} & =\text { heat flux }\left(\mathrm{W} / \mathrm{m}^{2}\right) \\
q^{\prime \prime \prime} & =\text { heat production per unit volume }\left(\mathrm{W} / \mathrm{m}^{3}\right) \\
R & =\text { thermal resistance }(\mathrm{K} / \mathrm{W}) \\
r & =\text { radius }(\mathrm{m}) \\
r & =\text { radial coordinate }(\mathrm{m}) \\
N^{2} &
\end{aligned}
$$




$$
\begin{aligned}
S & =\text { specific surface area }\left(\mathrm{m}^{-1}\right) \\
S_{\varnothing} & =\text { scalar source term } \\
T & =\text { temperature }(\mathrm{K}) \\
u & =\text { velocity }(\mathrm{m} / \mathrm{s}) \\
u_{o} & =\text { superficial gas velocity }(\mathrm{m} / \mathrm{s}) \\
V & =\text { volume }\left(\mathrm{m}^{3}\right) \\
v & =\text { kinematic viscosity }\left(\mathrm{m}^{2} / \mathrm{s}\right) \\
\vec{v} & =\text { velocity vector }(\mathrm{m} / \mathrm{s}) \\
z & =\text { axial coordinate }(\mathrm{m})
\end{aligned}
$$

Greek

$$
\begin{aligned}
\beta & =\text { thermal expansion coefficient }(1 / \mathrm{K}) \\
\beta_{f} & =\text { buoyancy force } \\
\Gamma & =\text { diffusion coefficient } \\
\Delta & =\text { increment } \\
\varepsilon & =\text { porosity } \\
\mu & =\text { dynamic viscosity }\left(\mathrm{N} \mathrm{s}^{-1} \mathrm{~m}^{-2}\right) \\
\rho & =\text { density }\left(\mathrm{kg} / \mathrm{m}^{3}\right) \\
\vec{\tau} & =\text { stress tensor }(\mathrm{Pa})
\end{aligned}
$$

\section{Subscript}

$$
\begin{aligned}
b & =\text { bed } \\
\text { eff } & =\text { effective } \\
f & =\text { fluid } \\
h & =\text { hydraulic } \\
i & =\text { interfacial } \\
p & =\text { particle } \\
\mathrm{p} & =\text { pressure } \\
R & =\text { radiated } \\
w & =\text { wall }
\end{aligned}
$$

Superscript

$$
\begin{aligned}
c & =\text { contact interface between particles } \\
f & =\text { free fluid } \\
i & =\text { interfacial } \\
p & =\text { porous medium }
\end{aligned}
$$

\section{Nondimensional numbers}

$$
\mathrm{Nu}_{w}=\text { Nusselt number, } \frac{q_{w} d_{h}}{k_{e f f}\left(T_{f}-T_{w}\right)}
$$

$$
\begin{aligned}
\operatorname{Pr} & =\text { Prandtl number, } \frac{\mu c_{p}}{k_{f}} \\
\mathrm{Ra} & =\text { Rayleigh number, } \frac{g \beta\left|T_{w}-T_{f}\right| d_{h}^{3}}{v^{2}} \operatorname{Pr}
\end{aligned}
$$

\section{Acknowledgments}

The authors would like to acknowledge the financial support from the Department of Mechanical and Aeronautical Engineering, University of Pretoria, Pretoria, South Africa.

\section{References}

1. D. MATZNER, "PBMR Project Status and the Way Ahead," Proc. 2nd Int. Topl. Mtg. HTR Technology, Beijing, China, September 2004.

2. J. SLABBER, "Technical Description of the PBMR Demonstration Power Plant, PBMR-016956," Rev. 4, Pebble Bed Modular Reactor (Pty) Ltd. (2006).

3. K. VAFAI, Handbook of Porous Media, 2nd ed., Taylor \& Francis, New York (2005).

4. D. A. NIELD and A. BEJAN, Convection in Porous Media, 3rd ed., Springer, New York (2006).

5. M. KAVIANY, Principles of Heat Transfer in Porous Media, 2nd ed., Springer-Verlag, New York (1995).

6. N. WAKAO and S. KAGUEI, Heat and Mass Transfer in Packed Beds, pp. 264-295, McGraw-Hill, New York (1982).

7. A. SHAMS et al., "Quasi-Direct Numerical Simulation of a Pebble Bed Configuration: Part 1: Flow (Velocity) Field Analysis," Nucl. Eng. Des., 263, 473 (2013); http://dx.doi. org/10.1016/j.nucengdes.2012.06.016.

8. F. AUGIER, F. IDOUX, and J. Y. DELENNE, "Numerical Simulations of Transfer and Transport Properties Inside Packed Beds of Spherical Particles," Chem. Eng. Sci., 65, 1055 (2010); http://dx.doi.org/10.1016/j.ces.2009.09.059.

9. M. NIJEMEISLAND and A. G. DIXON, "Comparison of CFD Simulations to Experiment for Convective Heat Transfer in a Gas-Solid Fixed Bed," Chem. Eng. J., 82, $231 \quad$ (2001); http://dx.doi.org/10.1016/S1385-8947 (00)00360-0.

10. CCM+ User Guide 7.02, Setting Material Properties Methods, p. 2463, CD-Adapco, Melville, New York (2013).

11. H. K. VERSTEEG and W. MALALASEKERA, An Introduction to Computational Fluid Dynamics: The Finite Volume Method, 2nd ed., Pearson Education Limited, United Kingdom (2007).

12. S. V. PATANKAR, Numerical Heat Transfer and Fluid Flow, Hemisphere Publishing Corporation, New York (1980). 
13. H. HUANG and J. AYOUB, "Applicability of the Forchheimer Equation for Non-Darcy Flow in Porous Media," SPE J., 13, 112 (2008); http://dx.doi.org/10.2118/ 102715-PA.

14. S. ERGUN, "Fluid Flow Through Packed Columns," Chem. Eng. Prog., 48, 89 (1959).

15. O. O. NOAH, J. F. SLABBER, and J. P. MEYER, "Natural Convection Heat Transfer Phenomena in Packed Bed Systems," Proc. Int. Mechanical Engineering Congress and Exposition, Montreal, Canada, 2014, ASME (2014).

16. S. P. TIMOSHENKO and J. N. GOODIE, Theory of Elasticity, Article 140, McGraw-Hill, New York (1970).

17. E. ACHENBACH, "Heat and Flow Characteristics of Packed Beds," Exp. Thermal Fluid Sci., 10, 17 (1995); http://dx.doi.org/10.1016/0894-1777(94)00077-L.

18. N. WAKAO, S. KAGUEI, and T. FUNAZKRI, "Effect of Fluid Dispersion Coefficients on Particle-to-Fluid Heat Transfer Coefficients in Packed Beds," Chem. Eng. Sci., 34, 325 (1978); http://dx.doi.org/10.1016/00092509(79)85064-2.

19. "Reactor Core Design of High-Temperature Gas-Cooled Reactors. Part 2: Heat Transfer in Spherical Fuel Elements," KTA3102.2, Nuclear Safety Standards Commission (1983).

20. A. G. DIXON, M. A. DiCOSTANZO, and B. A. SOUCY, "Fluid-Phase Radial Transport in Packed Beds of Low Tube-to-Particle Diameter Ratio," Int. J. Heat Mass Transfer, 27, 1701 (1984); http://dx.doi.org/10.1016/ 0017-9310(84)90153-4.

21. D. J. GUNN, "Transfer of Heat or Mass to Particle in Fixed and Fluidised Beds," Int. J. Heat Mass Transfer, 21, 467 (1978); http://dx.doi.org/10.1016/0017-9310(78)90080-7.

22. J. C. CHEN and S. W. CHURCHILL, "Radiant Heat Transfer in Packed Beds," AIChE J., 9, 35 (1963); http://dx.doi. org/10.1002/aic.690090108.
23. J. H. ROSENFELD, J. E. TOTH, and A. L. PHILLIPS, "Emerging Applications for Porous Media Heat Exchangers," Proc. Int. Conf. Porous Media and Their Applications in Science, Kona, Hawaii, 1996.

24. M. SUZUKI et al., "A Study on the Coordination Number in a System of Randomly Packed, Uniform-Sized Spherical Particles," Int. Chem. Eng., 21, 482 (1981).

25. K. MINATO et al., "Release Behavior of Metallic Fission Products from HTGR Fuel Particles at 1600 to $1900^{\circ} \mathrm{C}$," $J$. Nucl. Mater., 202, 47 (1993); http://dx.doi.org/10.1016/ 0022-3115(93)90027-V.

26. H. NICKEL et al., "Long Time Experience with the Development of HTR Fuel Elements in Germany," Nucl. Eng. Des., 217, 141 (2002); http://dx.doi.org/10.1016/S00295493(02)00128-0.

27. M. ISHIHARA et al., "Principle Design and Data of Graphite Components," Nucl. Eng. Des., 233, 1-3, 160 (2004); http://dx.doi.org/10.1016/j.nucengdes.2004.08.012.

28. T. IYOKUA et al., "Design of Core Components," Nucl. Eng. Des., 233, 1-3, 71 (2004); http://dx.doi.org/10.1016/j. nucengdes.2004.07.012.

29. K. KUGELER, "HTR Technology, NUCI 878 EB Study Guide," North West University, Potchefstroom, South Africa (2009).

30. A. KOSTER, H. D. MATZNER, and D. R. NICHOLSI, "PBMR Design for the Future," Nucl. Eng. Des., 222, 231 (2003); http://dx.doi.org/10.1016/S0029-5493(03)00029-3.

31. O. O. NOAH, J. F. SLABBER, and J. P. MEYER, "Experimental Evaluation of Natural Convection Heat Transfer in Packed Beds Contained in Slender Cylindrical Geometries," Proc. 5th Int. Conf. Applications of Porous Media, Cluj-Napoca, Romania, August 25-28, 2013, p. 301. 